\title{
Akıllı mobil telefonlardan elde edilen çift frekanslı ham GNSS gözlemleri ile PPP konum belirleme performansının araştırılması
}

\author{
Caneren Gül ${ }^{1 *}$ (D) Ali Hasan Doğan ${ }^{1}$ D , Taylan Öcalan ${ }^{1}$ (D) \\ ${ }^{1}$ Yıldız Teknik Üniversitesi, Davutpaşa Kampüsü, Inşaat Fakültesi, Harita Mühendisliği Bölümü, Esenler, İstanbul, Türkiye.
}

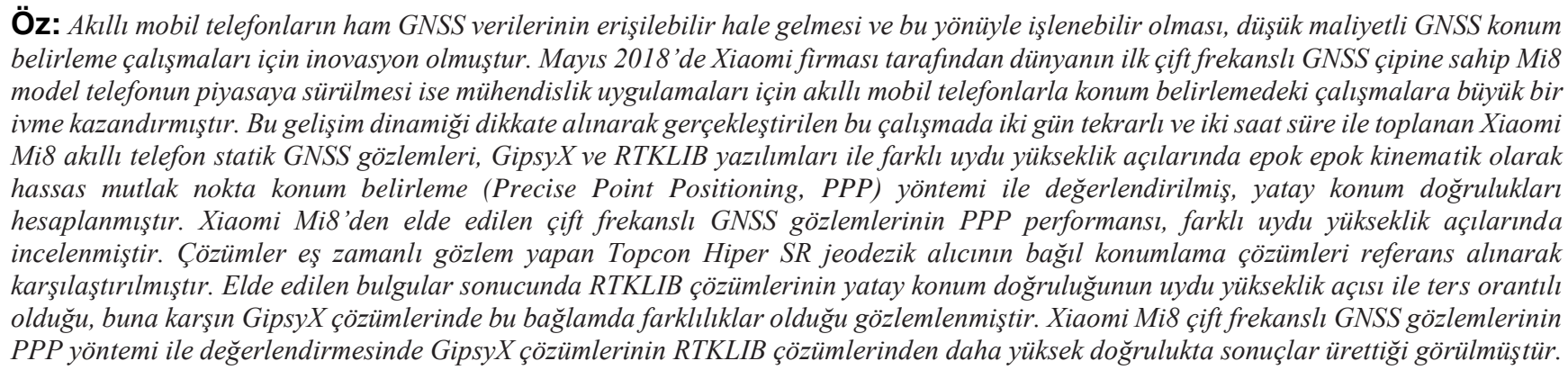

Anahtar Sözcükler: Akıllı mobil telefon, GNSS, PPP, Xiaomi Mi8, GipsyX, RTKLIB

\section{Investigation of PPP performance with dual frequency raw GNSS observations obtained from smartphones}

Abstract: Being accessible and processible of the raw GNSS data of smartphones has been an innovation for low-cost GNSS positioning studies. With the release of world's first smartphone with dual frequency GNSS chipset Mi8 by Xiaomi in May 2018, studies on smartphonebased positioning for engineering applications have gained great momentum. Considering these development dynamics, in this study, repeatedly collected Xiaomi Mi8 dual frequency static GNSS observations for two days in two-hour time span were processed using GipsyX and RTKLIB software with epoch by epoch kinematic Precise Point Positioning (PPP) method at different satellite elevation angles, and horizontal positioning accuracies were calculated. The PPP performance of dual frequency GNSS observations collected by Xiaomi Mi8 was investigated at different satellite elevation angles. The process outputs were compared with results analyzed by relative positioning method collected simultaneously with Topcon Hiper SR geodetic receiver by considering as a reference solution. According to the results, it was noted that the horizontal positioning accuracy of RTKLIB outputs has directly inverse relationship with increasing satellite elevation angles whereas GipsyX outputs have differences in this context. In the processing of Xiaomi Mi8 dual frequency observations with PPP method, it was concluded that GipsyX produced higher horizontal positioning accuracy than RTKLIB.

Keywords: Smartphone, GNSS, PPP, Xiaomi Mi8, GipsyX, RTKLIB 


\section{Giriş}

Günümüzde taşınabilir ve giyilebilir akıllı mobil cihazların (telefon, tablet, kol saati vb.) sayısı ve kullanım oranı her geçen gün artmaktadır. Bu cihazlar içerisinde akıllı telefonlar insanların günlük yaşantılarında kişisel asistanları olarak ön plana çıkmaktadırlar. Mobil internet erişimi sağlaması, sesli/görüntülü arama hizmetleri sunması, farklı sensör tabanlı uygulamaları gerçekleştirmesi, konumlama, navigasyon ve zamanlama (Positioning, Navigation and Timing, PNT) için hızlı çözüm üretmesi ve kolay taşınabilirliği dikkate alındığında akıllı telefonlar çok yönlü ve kullanışlı özelliklere sahiptir. Bu bakımdan akıllı telefonların ekonomik pazar büyüklüğü diğer mobil cihazlara göre oldukça fazladır. Avrupa GNSS Ajansı (European GNSS Agency, GSA) 2019 Pazar Raporu’na göre 2020 yılı sonu itibariyle akıllı telefon kullanıcılarının sayısının dünyada 3.5 milyara ulaşacağı tahmin edilmektedir (URL-1).

Akıllı telefonlarda farklı görevlere sahip birçok sensör bulunmaktadır. Bunlardan biri de farklı doğruluk seviyelerinde PNT hizmeti sağlayan gömülü GNSS çipleridir. PNT uygulamaları için ilk olarak 1999 yılında akıllı telefonlara GPS çipleri eklenmiştir. Günümüzde üretilen akıllı telefon konumlama çipleri ise GPS ile birlikte GLONASS, Galileo, BeiDou, QZSS ve SBAS sistemlerini destekler duruma gelmiştir (Banville \& Diggelen, 2016).

Mayıs 2016'da Google, “I/O 2016” konferansında Android 7.0 (Nougat) sürümü ile ak1llı cihazlarla toplanan ham GNSS verilerini kullanıcıların erişimine açacağını açıklamıştır. Bu karar, akıllı cihazlardaki ham GNSS verisinin işlenme sürecine müdahale olanağı sağlayarak, uydulara ait kod, taşıyıcı faz ve Doppler gözlemleri ile metre altı seviyede konum doğruluğu sağlamanın ilk önemli adımı olmuştur. Google’ın bu açıklamasından sonra Banville ve Diggelen (2016) çalışmalarında, Broadcom 4774 model gömülü GNSS çipe sahip Samsung Galaxy S7 marka akıllı telefonda L1 frekansında GPS, GLONASS, Galileo, BeiDou ve QZSS uydu sistemlerine ait kod, taşıyıcı faz ve Doppler gözlemlerini ham olarak kaydederek GNSS veri kalitesini incelemişlerdir. Sadece GPS gözlem verilerinin değerlendirildiği bu çalışmada, hassas konumlamada temel sorunun GNSS anten kalitesi ve sinyal sıçramaları olduğu vurgulanmıştır. Siddakatte, Broumandan ve Lachapelle (2017)'nin gerçekleştirdiği bir diğer çalışmada ise Broadcom 4774 model gömülü GNSS çipe sahip Huawei Mate 9 marka akıllı telefonda, dahili ve harici GNSS anten yapıları kullanılarak, ham ölçülerin kalitesi ve elde edilen konum doğruluklarının performansı çeşitli senaryolar altında test edilmiştir.

Akıllı telefonlar ile GNSS tabanlı hassas konum belirlemede ikinci önemli adım ise Mayıs 2018'de dünyanın ilk çift frekanslı telefonu olan Mi8'in, Xiaomi tarafından üretilmesi ve pazara sunulması ile gerçekleşmiştir. Xiaomi Mi8, GPS L1/L5 ve Galileo E1/E5a sinyallerini kaydedebilen Broadcom BCM47755 model gömülü GNSS çipine sahiptir. Özellikle akıllı telefonlar ile cm doğrulukta konum belirlemenin başlangıcını oluşturan bu gelişme, aynı zamanda GNSS'ye dayalı hassas konum belirleme çalışmalarında maliyetleri yüksek jeodezik alıcılar yerine, görece maliyetleri çok düşük olan akıllı telefonların mühendislik uygulamalarında kullanılabilirliği adına yapılacak birçok araştırmanın temel kaynağı ve motivasyonu olan süreci başlatmıştır (Robustelli, Baiocchi, \& Pugliano, 2019; Wu, Sun, Zhou, \& Zhang, 2019; Chen, Gao, Liu, \& Sun, 2019). 2018'de Xiaomi'den sonra Huawei, One Plus, Lenovo gibi farklı markalar da çeşitli modellerinde çift frekanslı GNSS çiplerine sahip yeni ürünlerini piyasaya sunmuşlardır.

Yüksek maliyetli jeodezik GNSS alıcıları ile düşük maliyetli akıllı telefonlar arasındaki en temel fark, kullanılan GNSS anten/çip özelliklerinden kaynaklanmaktadır. Akıllı telefon GNSS anten çipi, doğrusal (lineer) polarizasyona sahiptir. Bu durum antenin yakınındaki yüzeylerden yansıyan GNSS sinyallerinin çok yolluluk etkilerine duyarlı olmasına neden olur. Bu nedenle akıllı telefon GNSS anten çipi, çok yolluluk etkisini en aza indirmek için tasarlanan jeodezik GNSS alıcı antenine kıyasla düşük kaliteli GNSS ölçüleri toplar. Bu unsur akıllı telefon tabanlı GNSS konumlama için bir dezavantajdır 
(Robustelli vd., 2019; Zhang, Tao, Zhu, Shi, \& Whang, 2018; Pathak, Thornwall, Krier, \& Rowson, 2003). Buna karşın Mayıs 2016 tarihinde Google'ın akıllı telefonlarda ham GNSS ölçülerini kullanıcıların erişimine açması, ardından Mayıs 2018'de dünyanın ilk çift frekanslı telefonu olan Xiaomi Mi8'in pazara sunulmasıyla akıllı telefon tabanlı GNSS konumlama çalışmaları oldukça hız kazanmıştır.

Wu vd. (2019), RTKLIB ve GAMP yazılımlarını kullanarak Xiaomi Mi8 akıllı telefonunun konum belirleme performansını statik ve kinematik hassas nokta mutlak konumlama (Precise Point Positioning, PPP) yöntemi ile değerlendirmişlerdir. Değerlendirme sonucunda akıllı telefonun statik PPP konum belirleme performansının tek frekanslı jeodezik alıcıya yakın olduğu, kinematik PPP sonucunda ise yaklaşık 3-5 metre fark olduğu sonucuna ulaşmışlardır. Robustelli vd. (2019), Topcon GRS-1 jeodezik alıcı ve Topcon PG-A1 anten ile Xiaomi Mi8 akıllı telefonun konum belirleme performansını düşük ve yüksek çok yolluluk etkisine sahip ortamlarda kod ve taşıyıcı faz ölçüleri ile ayrı ayrı değerlendirmişlerdir. Çalışmada TEQC (Estey \& Meertens, 1999) yazılımı kullanılarak kod ölçülerinin çok yolluluk etkisi kestirilmiş, ayrıca 3600 epoğa sahip 1 saatlik verinin 900 epoğunda (\%25'inde) yüksek çok yolluluk etkisi gözlemlenmiştir. Akıllı telefon ile toplanan verinin, jeodezik alıcıya göre daha düşük taşıyıcı gürültü oranı (Carrier-to-Noise Ratio, $\mathrm{C} / \mathrm{N}_{0}$ ) profiline sahip olduğu ortaya konmuştur. Jeodezik alıcı referans alındığında akıllı telefon GNSS ölçüleri ile düşük çok yolluluk etkisine sahip ortamda 1.02 m, yüksek çok yolluluk etkisine sahip ortamda ise 1.95 m doğruluk elde edilmiştir. Kulikov, Chugunov ve Zamolodchikov (2019), araç ve yaya navigasyonunda sıklıkla yapılan manevralar esnasında jeodezik alıcı ve Samsung Galaxy S8 akıllı telefon ile veriler elde etmişlerdir. Veriler ölçü sonrası değerlendirilmiş ve elde edilen sonuçlar jeodezik alıcı ile elde edilen sonuçlarla karşılaştırılmıştır. Değerlendirme sonucunda akıllı telefon çözümleri ile jeodezik alıcı çözümleri arasında konum değerleri farkının 1-3 m arasında değiştiği gösterilmiştir. Elmezayen ve El-Rabbany (2019), Trimble R9 jeodezik alıcı ve Xiaomi Mi8 akıllı telefon performansını statik ve kinematik ölçümlerle değerlendirerek karşılaştırmıştır. Trimble Business Center (TBC) yazılımı kullanılarak bağıl çözümler yapılmış, ardından geliştirilen özel bir yazılım ile PPP çözümleri gerçekleştirilmiştir. PPP çözümleri hem gerçek zamanlı (NAVCAST servisi ile) elde edilmiş, hem de sonradan değerlendirilmiştir. PPP çözümleri karşılaştırıldığında gerçek zamanlı ve sonradan değerlendirilen statik çözümlerde desimetre mertebesinde doğruluk elde edilmiştir. Kinematik çözümlerle elde edilen doğruluk ise metre mertebesindedir. Aggrey, Bisnath, Naciri, Shinghal ve Yang (2019), SwiftNav Piksi ve Topcon NET-G3A jeodezik alıcılar ile Xiaomi Mi8, Google Pixel 3, Huawei Mate 20 ve Samsung Galaxy S9 cihazlarının çoklu-GNSS PPP performanslarını değerlendiren bir çalışma yapmışlardır. Çalışmada alıcılarla statik durumda 6 saat gözlem yapılmıştır. Ayrıca, aracın üstüne yerleştirilen SwiftNav Piksi alıcı ve aracın içinde ön kısma kurulan akıllı telefonlar ile önceden belirlenen bir güzergâh üzerinde kinematik ölçümler gerçekleştirilmiştir. Değerlendirme sonucunda akıllı telefonlarda, SwiftNav Piksi ve Topcon NET-G3A alıcılarına göre daha yüksek çok yolluluk etkisi ve daha düşük C/N0 tespit edilmiştir. Değerlendirmeler sonucunda çift frekanslı alıcıya sahip telefonlarda, 38 dakikalık gözlemle statik modda desimetre mertebesinde doğruluk, kinematik modda ise birkaç metre mertebesinde doğruluk elde etmenin mümkün olduğu belirtilmiştir.

Akıllı telefonlarda devrim niteliğindeki bu gelişmeler, kişisel uygulamaların yanında yakın gelecekte birçok mühendislik çalışması için de bu cihazların kullanım potansiyelini artırmıştır. Öyle ki akıllı telefonlarda kullanılan GNSS çipi kalitesi ve özellikleri artarken, aynı zamanda 3 boyutlu modelleme çalışmaları için lazer tarama sensör entegrasyonları da sağlanmaya başlamıştır. $\mathrm{Bu}$ gelişim dinamikleri dikkate alınarak gerçekleştirilen bu çalışmada, akıllı telefonlardan elde edilen çift frekanslı ham GNSS gözlemlerinin PPP performansı araştırılmıştır. Bu kapsamda Xiaomi Mi8 akıllı telefon ve Topcon Hiper SR jeodezik alıcı ile ardışık iki günde ikişer saatlik statik GNSS gözlemleri yapılmıştır. Xiaomi Mi8 ve Topcon Hiper SR' den eş zamanlı olarak elde edilen statik GNSS gözlemleri, farklı yükseklik açıları seçilerek PPP tekniği ile epok epok kinematik olarak analiz edilmiştir. Veri değerlendirme ve analiz aşamalarında hem RTKLIB hem de GipsyX yazılımları kullanılmıştır. Elde edilen tüm sonuçlar jeodezik alıcı bağıl konum belirleme çözüm sonuçları ile karşılaştırılmıştır. 


\section{PPP Tekniği}

PPP tekniği, 1990'ların sonunda bağıl konum belirleme tekniğine alternatif olarak geliştirilmiş, tek bir GNSS alıcısının kullanıldığ özel bir mutlak konum belirleme tekniğidir (Zumberge, Heflin, Jefferson, Watkins, \& Web, 1997). PPP yönteminin gerek ölçü sonrası değerlendirme, gerekse gerçek zamanlı konumlama için sağladığı birçok avantaj bulunmaktadır. Tek bir GNSS alıcısının kullanılması, maliyetin görece düşük olması, referans istasyonlara gereksinim duyulmaması bunların başlıcalarıdır. Bu yönüyle bağıl/diferansiyel konum belirlemeye önemli bir alternatif oluşturmaktadır (Erdoğan, Karlıtepe, Öcalan, \& Tunalıoğlu 2018; Öcalan, Erdoğan, Tunalıoğlu, \& Durdağ 2016). Bu nedenle, statik ve kinematik modda gerçekleştirilen GNSS uygulamalarında cm-dm seviyesinde doğruluk sağlayan PPP tekniği büyük ilgi görmüş ve yaygın olarak kullanılmaya başlanmıştır. PPP tekniği, GNSS tabanlı konumlamada jeodezik-jeofizik ölçme uygulamalarında, iklim bilimi çalışmalarında, kıyı mühendisliğinde, hidrografik-oşinografik çalışmalarda, doğal afetlerin izlenmesi ve atmosferik araştırmalarda, tarım, madencilik, inşaat, enerji (petrol ve gaz) uygulamalarında yaygın olarak kullanılmaktadır (Lipatnikov \& Shevchuk, 2019; Erdoğan vd., 2018). Yakın gelecekte ise özellikle araç park etme, kargo teslimatı, ortak araç kullanımı, acil durum müdahalesi ve otonom sürüş gibi akıllı şehir uygulamalarında da etkin olarak kullanımı beklenmektedir (Robustelli vd., 2019).

GNSS gözlemleri için çift frekanslı iyonosfer-bağımsız doğrusal kombinasyonun kullanımı, uydu ve alıcı kaynaklı hatalar ve jeofiziksel etkilerin modellenmesi temel olarak geleneksel PPP'yi tanımlamaktadır (Aggrey vd., 2019; Chen \& Gao, 2005; Héroux vd., 2004; Zumberge vd., 1997). Genel olarak PPP'de kod (1) ve taşıyıcı faz (2) gözlem eşitlikleri aşağıdaki şekildedir.

$P_{r}^{s}=\rho_{r}^{s}+c\left(d T_{r}-d T^{s}\right)+d_{\text {orb }}+d_{\text {tro }}+d_{i o n}^{r s}+\varepsilon_{P}^{r s}$

$\phi_{r}^{s}=\rho_{r}^{s}+c\left(d T_{r}-d T^{s}\right)+d_{o r b}+d_{t r o}-d_{i o n}^{r s}+N^{r s}+\varepsilon_{\phi}^{r s}$

Burada alt indis $r$ ve $s$ sirasıly alıcı ve uydu, $P_{r}^{s}$ ve $\phi_{r}^{s}$ sirasıyla kod ve faz gözlemleri, $\rho_{r}^{s}$ alıcı ve uydu arasındaki geometrik mesafe, $c$ ışı hızı, $d T_{r}$ ve $d T^{s}$ sırasıyla alıcı ve uydu saat hataları, $d_{\text {orb }}$ uydu yörünge hataları, $d_{\text {tro }}$ ve $d_{i o n}^{r s}$ sırasıyla troposfer ve iyonosfer gecikmesi, $N^{r s}$ faz belirsizliği, $\varepsilon_{P}^{r s}$ ve $\varepsilon_{\phi}^{r s}$ ssrasıyla kod ve faz gözlemlerine ait diğer (okyanus ve karasal yükleme, sinyal kod kayıklığı-bias, yer dönüklüğü, uydu faz dönmesi, çok yolluluk ve anten düzeltmeleri vs.) hatalardır. İlk mertebeden iyonosferik etkiler iyonosfer-bağımsız gözlem kombinasyonları ile, yüksek mertebeden iyonosferik etkiler, uydu yörünge ve saat hataları, jeofiziksel etkiler, sinyal kod kayıklığı (Differential Code Bias, DCB) ve anten düzeltmeleri GNSS analiz merkezleri tarafindan sağlanan ilgili ürünler ile modellendiğinde (1) ve (2) eşitlikleri, kod ve faz ölçüleri için iyonosferbağımsız olarak (3) ve (4) eşitliklerine sadeleştirilebilir (Chen vd., 2019).

$P_{r}^{s}=\rho_{r}^{s}+c d T_{r}+d_{t r o}+\varepsilon_{P}^{r s}$

$\phi_{r}^{s}=\rho_{r}^{s}+c d T_{r}+d_{t r o}+N^{r s}+\varepsilon_{\phi}^{r s}$

\section{Uygulama}

\subsection{Test Noktası}

Xiaomi Mi8 akıllı telefondan elde edilen çift frekanslı ham GNSS gözlemlerinin PPP konum belirleme performansının araştırılması için Yıldız Teknik Üniversitesi Davutpaşa Kampüsü İnşaat Fakültesi çatısında bulunan ERBD pilyesi test noktası olarak seçilmiştir (Şekil 1). ERBD test noktası yakın çevresinde yapı, ağaç, yansıtıcı yüzey vb. bulunmaması nedeniyle, sinyal çok yolluluk etkisini en aza indirme özelliğine sahip olduğundan uygulama için tercih edilmiştir. 
28-29 Ocak 2020 tarihlerinde iki gün süreyle ERBD noktasında aynı zaman aralığında Topcon Hiper SR ve Xiaomi Mi8 ile 2 saat süreyle eş zamanlı statik GNSS gözlemleri gerçekleştirilmiştir (Şekil 1). Xiaomi Mi8 ve Topcon Hiper SR için veri kayıt aralıkları $1 \mathrm{~Hz}$ olarak seçilmiş, uydu yükseklik açıları ise $0^{\circ}$ olarak belirlenmiştir.

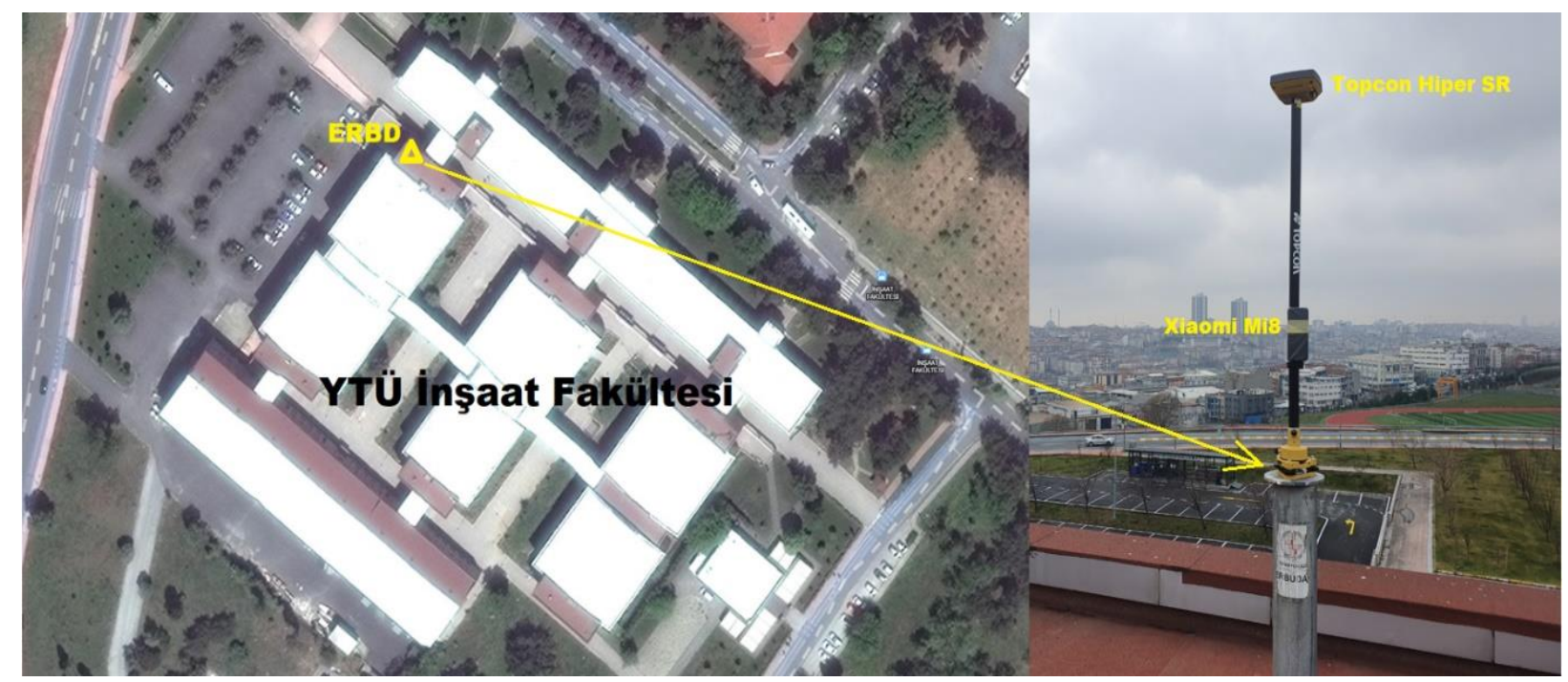

Şekil 1: ERBD test noktasında Topcon Hiper SR ve Xiaomi Mi8 akıllı telefonun eş zamanlı statik GNSS ölçümü

Gerçekleştirilen deney sonucunda ilgili verilerin RTKLIB ve GipsyX yazılımlarında değerlendirilmesi ve analizi için tüm GNSS gözlemleri RINEX formatında elde edilmiştir. Xiaomi Mi8 ham GNSS gözlemlerinin elde edilmesinde Geo++ RINEX Logger yazılımından yararlanılmıştır (URL-2).

\subsection{Verilerin Değerlendirilmesi ve Analizi}

İki gün tekrarlı ve 2 saat olarak elde edilen Topcon Hiper SR ve Xiaomi Mi8 statik GNSS gözlemlerinin veri değerlendirme ve analiz aşamasında kullanılan uydu sistemleri, sinyaller, yöntemler ve yazılımlar Tablo 1'de gösterilmiştir.

Tablo 1: Veri değerlendirme ve analizlerde kullanılan uydu sistemleri, sinyaller, yöntemler ve yazılımlar

\begin{tabular}{ccccc}
\hline Cihaz & Uydu Sistemleri & Sinyaller & Değerlendirme Yöntemi & Yazılım \\
\hline \multirow{2}{*}{ Topcon Hiper SR } & GR & L1 - L2 & Bağ1l & $\begin{array}{l}\text { Topcon MAGNET } \\
\text { Tools }\end{array}$ \\
\hline \multirow{2}{*}{ Xiaomi Mi8 } & GE & L1 - L5 & PPP & RTKLIB v2.4.2 ve \\
& & E1 - E5a & GipsyX v1.2 \\
\hline & $(G): G P S$ & $(R): G L O N A S S$ & $(E):$ Galileo &
\end{tabular}

Referans çözüm olarak kullanılacak olan Topcon Hiper SR jeodezik alıcısının GPS+GLONASS çift frekans gözlemleri, bağıll konum belirleme yöntemiyle Topcon MAGNET Tools yazılımıyla değerlendirilmiştir. Değerlendirmede İstanbul'da yerel düzeyde faaliyet gösteren İSKİ-UKBS ağının PALA ve KCEK istasyon koordinatları sabit alınarak gözlem epoğunda ve ITRF2014 datumunda Topcon Hiper SR jeodezik alıcısının anten faz merkezi yatay koordinatları toposentrik koordinat sisteminde $(n, e)$ hesaplanmıştır.

Xiaomi Mi8 akıllı telefonundan elde edilen çift frekans gözlemleri GPS+Galileo sistemlerine aittir. Xiaomi Mi8 gözlemlerinin değerlendirme aşamasında PPP yöntemi kullanılmıştır. 2 saat süreli ve 2 gün tekrarlı olarak elde edilen bu gözlemler hem RTKLIB hem de GipsyX yazılımları ile epok epok (1 sn aralıklı) kinematik olarak değerlendirilmiştir. 
RTKLIB ve GipsyX yazılımları ile epok epok kinematik olarak analiz edilen Xiaomi Mi8 verilerinin PPP çözümleri için kullanılan ürünler ve değerlendirme stratejileri Tablo 2'de yer almaktadır.

Tablo 2: RTKLIB ve GipsyX'de kullanılan ürün ve stratejiler

\begin{tabular}{llc}
\hline Parametre & RTKLIB & GipsyX \\
\hline Yayın yörünge bilgisi & IGS & CODE \\
\hline Hassas yörünge bilgisi & CODE & CODE \\
\hline Saat bilgisi & CODE & CODE \\
\hline TEC & CODE & Modellendi \\
\hline ZWD & Modellendi & Kismi (Float) \\
\hline Faz Belirsizliği Çözümü & & $0^{\circ}, 15^{\circ}, 30^{\circ}$ ve $45^{\circ}$ \\
\hline Uydu Yükseklik Açısı & & \\
\hline
\end{tabular}

GipsyX ve RTKLIB yazılımlarında Avrupa Yörünge Belirleme Merkezi (Center for Orbit Determination in Europe, CODE) tarafından sağlanan hassas uydu yörünge ve saat bilgileri kullanılmıştır. RTKLIB değerlendirme aşamasında yayın yörünge bilgilerine de ihtiyaç duymaktadır. Bu bilgi Uluslararası GNSS Servisi (International GNSS Service, IGS) tarafından MultiGNSS Experiment (MGEX) projesi kapsamında üretilen ürünler yardımıyla sağlanmıştır. Her iki yazılımda da iyonosferden bağımsız (IonoFree) sinyaller değerlendirilmiştir. Başlangıç tamsayı faz belirsizliği kısmi olarak belirlenmiştir. DCB ve yer dönmesi dikkate alınmıştır. Troposferin ıslak bileşenine ait etki modellenerek giderilmiştir. RTKLIB yazılımına ait detaylı bilgi Takasu (2013)'de, GipsyX'e ait detaylı bilgi ise Bertiger vd. (2020)'de bulunabilir.

Xiaomi Mi8 akıllı telefon GNSS gözlemleri $0^{\circ}$ uydu yükseklik açısında elde edilmiştir. Akıllı mobil telefonların GNSS anten çipi özellikleri dikkate alındığında sinyal çok yolluluk etkisinin konum belirleme performasını belirgin düzeyde etkilediği bilindiğinden, PPP analizleri $0^{\circ}, 15^{\circ}, 30^{\circ}$ ve $45^{\circ}$ için farklı uydu yükseklik açılarına göre yapılarak dört farklı çözüm elde edilmiştir. PPP konum belirleme çözümlerinde önemli bir dezavantaj tamsayı belirsizliği çözüm süresinin uzun olmasıdır. Xiaomi Mi8 akıllı telefonunun gerek RTKLIB gerekse GipsyX yazılımlarında gerçekleştirilen epok epok kinematik PPP analizlerinde faz belirsizliği çözüm durumu kısmi (float) olarak belirlenmiştir.

Topcon Hiper SR alıcısından elde edilen bağıl çözüm koordinatları referans alınarak GipsyX ve RTKLIB yazılımları ile epok epok elde edilen kinematik PPP çözümleri toposentrik koordinat sistemine dönüştürülmüştür. Çözümlerin karesel ortalama hatas1 $(\mathrm{KOH})$ :

$K O H_{i}=\sqrt{\frac{\sum_{k}^{m} \Delta_{i, k}^{2}}{m}}, i \in\{n, e\}, k \in\{1,2, \ldots, m\}$

eşitliği ile elde edilmiştir. Eşitlikte kullanılan $\Delta$ : koordinat değeri (referans koordinatlardan farklar), i: koordinat bileşen indisi, $n$ : toposentrik kuzey, $e$ : toposentrik doğu ve $m$ : ölçü sayısıdır.

\subsection{Bulgular}

Xiaomi Mi8 akıllı telefonundan 28-29 Ocak 2020 (day of year-DoY: 28 ve 29) tarihlerinde elde edilen 2 saat süreli çift frekanslı GPS+Galileo gözlemleri RTKLIB ve GipsyX yazılımlarıyla $0^{\circ}, 15^{\circ}, 30^{\circ}$ ve $45^{\circ}$ uydu yükseklik açıları ile değerlendirilmiş ve Bölüm 3.2'de açıklandığı üzere $n, e$ bileşenlerine ait KOH değerleri hesaplanmıştır (Tablo 3). Tablo 4 ve Şekil 2 ise $0^{\circ}, 15^{\circ}, 30^{\circ}$ ve $45^{\circ}$ uydu yükseklik açıları için GipsyX ve RTKLIB'den elde edilen yatay konum doğruluklarını göstermektedir. 
Tablo 3: Xiaomi Mi8 için GipsyX ve RTKLIB çözümlerinden elde edilen KOH değeri sonuçları

\begin{tabular}{|c|c|c|c|c|c|}
\hline \multirow[t]{2}{*}{ КОН } & \multicolumn{2}{|c|}{$\begin{array}{c}28 \text { Ocak } 2020 \\
\text { DoY:28 }\end{array}$} & \multicolumn{2}{|c|}{$\begin{array}{c}29 \text { Ocak } 2020 \\
\text { DoY:29 } \\
\end{array}$} & \multirow{2}{*}{ Yükseklik Açısı ( $\left.{ }^{\circ}\right)$} \\
\hline & GipsyX & $R T K L I B$ & GipsyX & $R T K L I B$ & \\
\hline \multirow{4}{*}{$n(m)$} & 3.17 & 5.19 & 3.54 & 6.15 & 0 \\
\hline & 2.58 & 6.10 & 4.17 & 6.76 & 15 \\
\hline & 2.91 & 6.22 & 3.95 & 8.36 & 30 \\
\hline & 9.30 & 6.57 & 2.54 & 33.34 & 45 \\
\hline \multirow{4}{*}{$e(m)$} & 2.04 & 4.96 & 3.71 & 6.47 & 0 \\
\hline & 2.15 & 5.42 & 4.03 & 6.65 & 15 \\
\hline & 5.20 & 5.93 & 5.89 & 6.49 & 30 \\
\hline & 4.70 & 6.74 & 2.87 & 11.50 & 45 \\
\hline
\end{tabular}

n ve e için $\mathrm{KOH}$ değerleri ile yatay konum doğruluğu eşitlik (6) ile hesaplanır.

Yatay Konum Doğruluğ $=\sqrt{K O H_{n}^{2}+K O H_{e}^{2}}$

Tablo 4: Xiaomi Mi8 için GipsyX ve RTKLIB çözümlerinden elde edilen yatay konum doğruluğu sonuçları

\begin{tabular}{cccccc}
\hline & \multicolumn{2}{c}{$\begin{array}{c}\text { 28 Ocak 2020 } \\
\text { DoY:28 }\end{array}$} & \multicolumn{2}{c}{$\begin{array}{c}\text { Ocak 2020 } \\
\text { DoY:29 }\end{array}$} & \multirow{2}{*}{$\begin{array}{c}\text { Yükseklik Açısı } \\
\text { () }\end{array}$} \\
\cline { 2 - 5 } & GipsyX & RTKLIB & GipsyX & RTKLIB & \\
\hline & 3.8 & 7.2 & 5.1 & 8.9 & 0 \\
\cline { 2 - 5 } $\begin{array}{c}\text { Yatay Konum } \\
\text { Doğruluğu(m) }\end{array}$ & 3.4 & 8.2 & 5.8 & 9.5 & 15 \\
\cline { 2 - 5 } & 6.0 & 8.6 & 7.1 & 10.6 & 30 \\
\cline { 2 - 5 } & 10.4 & 9.4 & 3.8 & 35.3 & 45 \\
\hline
\end{tabular}

Xiaomi Mi8 akıllı telefon çift frekanslı GPS+Galileo gözlemleri için 28. günde GipsyX yazılımında yatay konum doğruluğu için elde edilen en iyi değer $15^{\circ}$ yükseklik açısında $3.4 \mathrm{~m}$ iken en kötü değer $45^{\circ}$ yükseklik açısında 10.4 m' dir. 29 . günde ise en iyi değer $45^{\circ}$ yükseklik açısında $3.8 \mathrm{~m}$ iken en kötü değer $30^{\circ}$ yükseklik açısında $7.1 \mathrm{~m}$ olarak hesaplanmıştır.

RTKLIB yazılımında en iyi değer $0^{\circ}$ yükseklik açısında 28. ve 29. günlerde sırasıyla $7.2 \mathrm{~m}$ ve $8.9 \mathrm{~m}$ hesaplanmıştır. En kötü değer ise $45^{\circ}$ yükseklik açısında 28. ve 29. günlerde sırasıyla $9.4 \mathrm{~m}$ ve $35.3 \mathrm{~m}$ hesaplanmıştır. 28. ve 29. günler için sırasıyla GipsyX ve RTKLIB çözümleri Şekil 3 ve Şekil 4’te görselleştirilmiştir. 

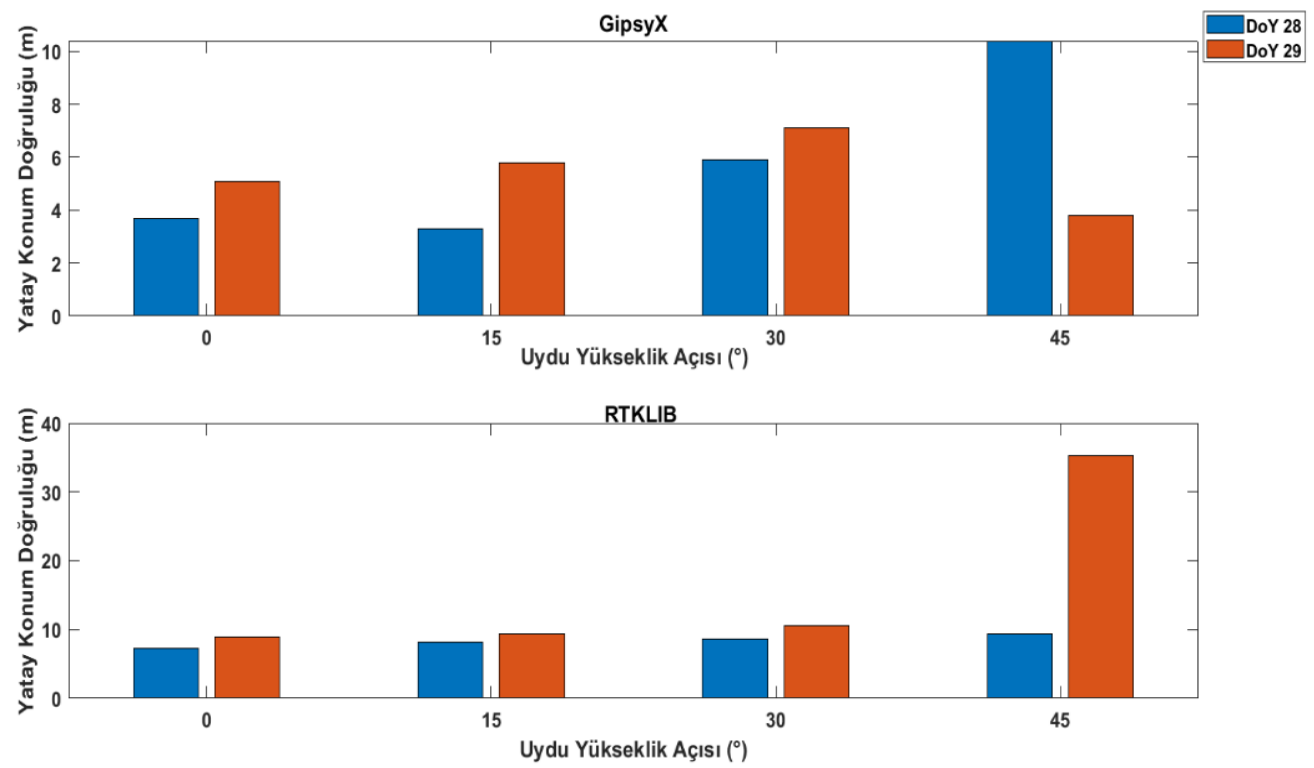

Şekil 2: GipsyX ve RTKLIB yatay konum doğrulukları(m)
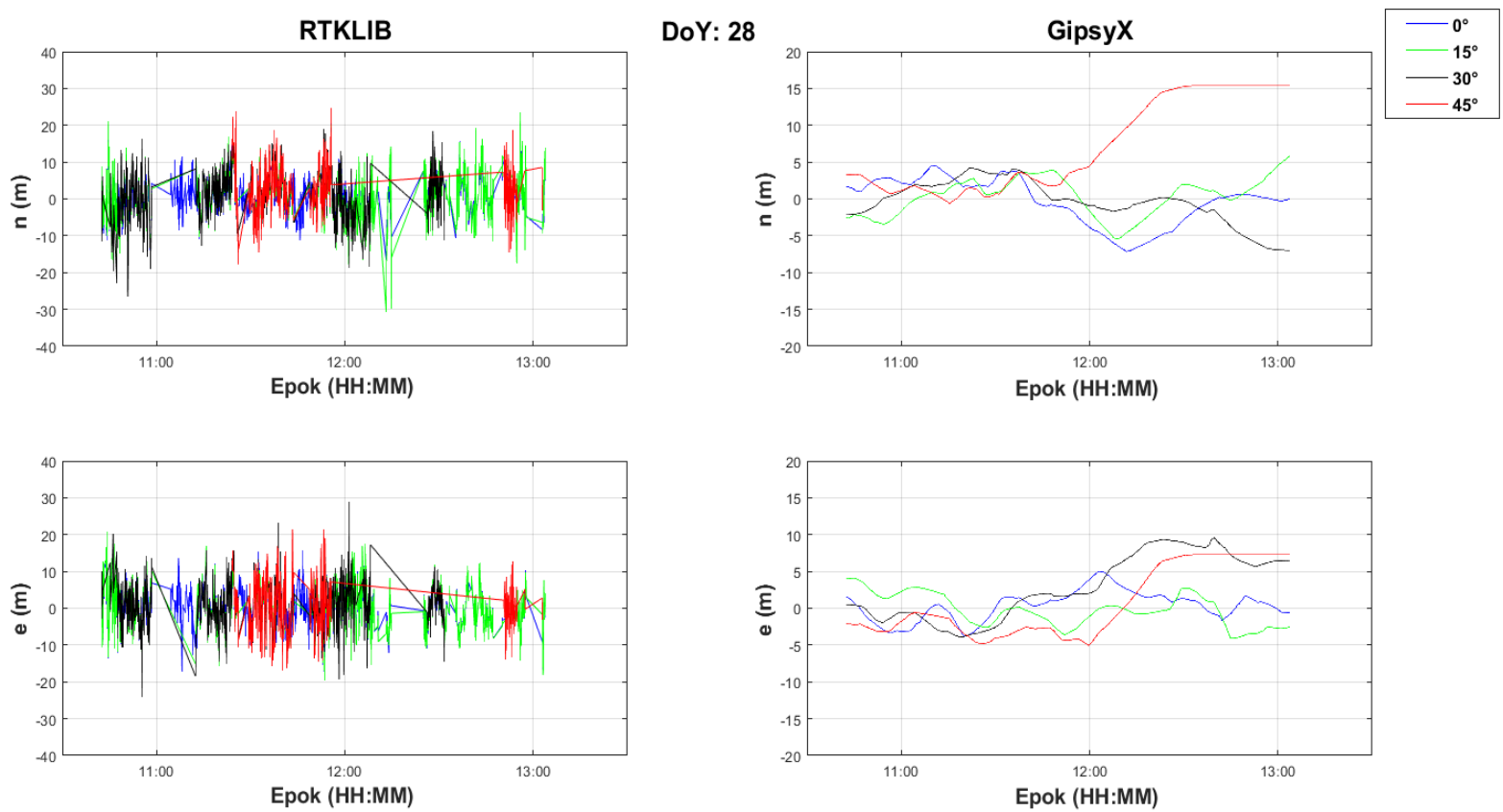

Şekil 3: 28. gün RTKLIB ve GipsyX çözümleri

RTKLIB, aynı epokta aynı uydudan çift frekans gözlem bulunmadığı durumda ilgili epoktaki ölçüleri değerlendirmeye almamaktadır. Ancak GipsyX rastgele yürüyüş modeli ile gözlemlerin bulunmadığı epoklarda da koordinat üretebilmektedir. Bu bağlamda ilgili yazılımların çözüm algoritma yaklaşımları farklılık taşımaktadır. 


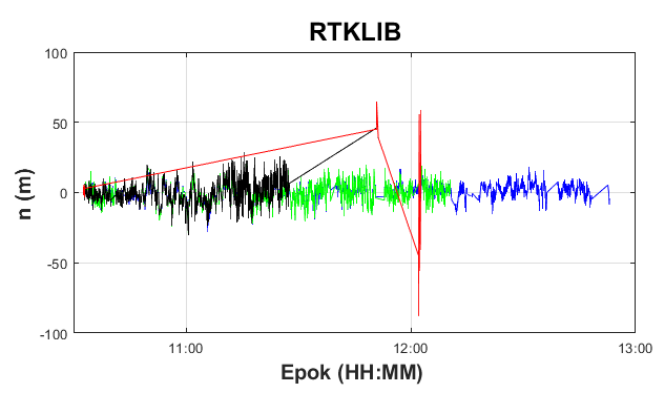

DoY:29
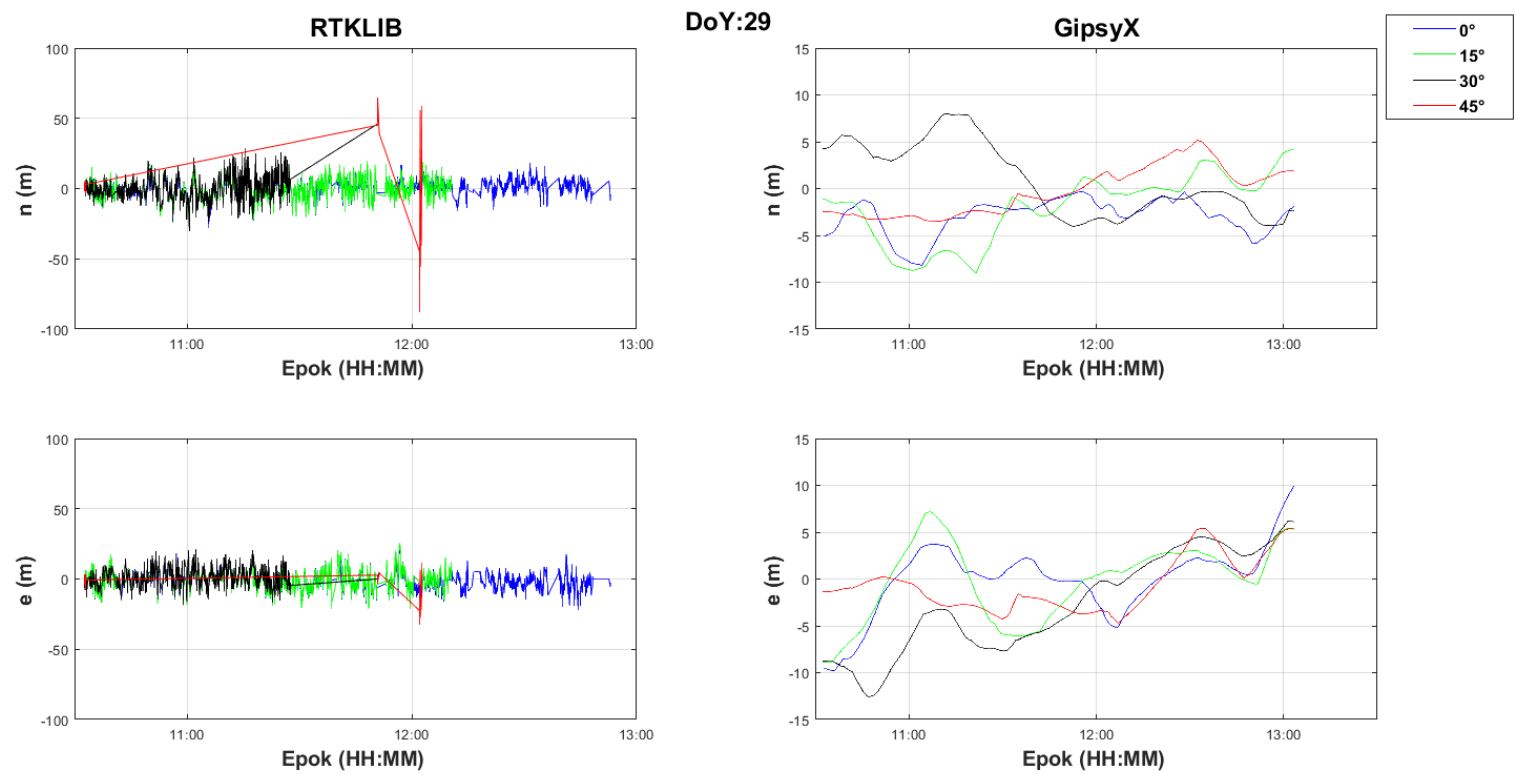

Şekil 4: 29. gün RTKLIB ve GipsyX çözümleri

Uydu yükseklik açısı arttıkça gözlenen uydu sayısı azalmakta, dolayısıyla değerlendirmeye alınan ölçü sayısı da azalmaktadır. RTKLIB'de özellikle $30^{\circ}$ ve $45^{\circ}$ uydu yükseklik açılarında önemli derecede ölçü kaybı görülmektedir. Öyle ki, 29. günde $45^{\circ}$ uydu yükseklik açısında toplam gözlem epoğunun yalnızca \%0.4'ü değerlendirmeye alınabilmiş ve çok düşük doğruluk elde edilmiştir. RTKLIB yazılımı kullanıldığında uydu yükseklik açılarına göre değerlendirmeye alınan gözlem epoğu oranları 28. gün ve 29. gün için Tablo 5’te gösterilmiştir.

Tablo 5: 28. ve 29. gün Xiaomi Mi8 RTKLIB çözümlerinde uydu yükseklik açısına göre değerlendirilmeye alınan epok oranları

\begin{tabular}{ccc}
\hline \multirow{2}{*}{ Yükseklik Açısı ( $\left.{ }^{\circ}\right)$} & Değerlendirmeye Alınan Epok Oranı (\%) \\
\cline { 2 - 3 } & 28. gün & 29. gün \\
\hline 0 & 41.2 & 52.6 \\
\hline 15 & 38.9 & 45.3 \\
\hline 30 & 31.0 & 27.6 \\
\hline 45 & 12.9 & 0.4 \\
\hline
\end{tabular}

\section{Sonuçlar}

Akıllı mobil telefonlardan ham GNSS gözlem verilerinin elde edilmesi, bu cihazların mühendislik uygulamalarındaki kullanım potansiyelini artırmıştır. Son yıllarda çift frekanslı GNSS sinyal çipine sahip akıllı mobil telefonların sayısının artması da, bu sürecin gelişmesine katkı sağlamıştır. Özellikle cm-dm doğruluklarında konum belirleme için akıllı mobil telefonların kullanım potansiyelini araştıran çalışmaların sayısı gün geçtikçe artmaktadır. PPP tekniği ise zaman, maliyet ve işgücü kriterleri açısından bağıl konum belirlemeye önemli bir alternatif olarak GNSS konumlamada kullanıcılar tarafindan tercih edilme oranı giderek artan güncel bir yöntem olarak ön plana çıkmaktadır.

Bu gelişim dinamikleri dikkate alınarak gerçekleştirilen çalışmada, dünyanın ilk çift frekanslı akıllı mobil telefonu olma özelliğine sahip Xiaomi Mi8 ile 2 gün tekrarlı olarak 2 saatlik statik GNSS gözlemleri yapılmıştır. Elde edilen gözlemler, farklı uydu yükseklik açıları ile RTKLIB ve GipsyX yazılımları ile epok epok kinematik modda PPP tekniği ile 
değerlendirilmiştir. Değerlendirme sonuçları, Topcon Hiper SR jeodezik alıcısı ile elde edilen bağıl çözümler referans alınarak karşılaştııılmıştır. Farklı çözüm algoritmaları kullanan RTKLIB ve GipsyX yazılımlarında, değişen uydu yükseklik açısının çözümlere etkisi incelenmiştir. Xiaomi Mi8 çift frekans GPS+Galileo gözlemlerinde GipsyX ile elde edilen çözümlerde en iyi yatay konum doğruluğu 28. günde $15^{\circ}$ yükseklik açısı ile, 29. günde ise $45^{\circ}$ yükseklik açısı ile elde edilmiştir. Diğer yandan RTKLIB'de, uydu yükseklik açısı arttıkça yatay konum doğruluğu belirgin düzeyde olumsuz etkilenmektedir. En iyi yatay konum doğruluğu her iki günde de $0^{\circ}$ yükseklik açısı ile elde edilmiştir. GipsyX için, iki günlük tekrarlı olarak yapılan değerlendirme sonuçları incelendiğinde, uydu yükseklik açısı ile yatay konum doğrulukları arasında doğrudan bir ilişki tanımlanamamaktadır. Ancak RTKLIB yazılımında uydu yükseklik açısının arttııılmasının elde edilen yatay konum doğruluğunu olumsuz etkilediği açıkça görülebilmektedir. Uydu yükseklik açısının artırılmasıyla azalan gözlem sayısının etkisi, RTKLIB değerlendirme sonuçlarında daha baskın hale gelmektedir. Tüm bu sonuçların yanında GipsyX ile elde edilen en iyi yatay konum doğruluğu ile RTKLIB'den elde edilen en iyi yatay konum doğruluğu arasındaki fark yaklaşık 4 m’dir. Sonuç olarak Xiaomi Mi8 ile 2 gün tekrarlı ve 2 saatlik ölçü süresine sahip çift frekanslı GNSS gözlemlerinin PPP analizlerinde, farklı uydu yükseklik açıları için GipsyX ile elde edilen yatay konum doğruluklarının RTKLIB'e göre görece daha iyi sonuçlar verdiği gözlemlenmiştir.

\section{Teşekkür}

Verilerin değerlendirilmesinde kullanılan RTKLIB yazılımı için Tomoji Takasu'ya, GipsyX yazılımı için NASA-JPL'ye, ayrıca veri ve ürün desteklerinden ötürü IGS, CODE ve İSKİye teşekkür ederiz.

\section{Yazar Katkısı}

Caneren Gül: Tasarım, Literatür taraması, Analiz ve yorumlama, Yazım, Veri toplama ve işleme, Makale değerlendirme. Ali Hasan Doğan: Denetleme, Yazım, Literatür taraması, Analiz ve yorumlama, Veri toplama ve işleme, Makale değerlendirme. Taylan Öcalan: Fikir, Tasarım, Analiz ve yorumlama, Denetleme, Yazım, Makale değerlendirme.

\section{Çıkar Çatışması Beyanı}

Yazarlar, bu çalı̧̧mada bilinen ilgili herhangi bir finansal veya finansal olmayan çıkar çatışması olmadığını beyan eder.

\section{Kaynaklar}

Aggrey, J., Bisnath, S., Naciri, N., Shinghal, G., \& Yang, S. (2019). Use of PPP processing for next-generation smartphone GNSS chips: key benefits and challenges. Proceedings of the 32nd International Technical Meeting of the Satellite Division of The Institute of Navigation (ION GNSS+ 2019), 3862-3878.

Banville, S., \& Van Diggelen, F. (2016). Precise positioning using raw GPS measurements from Android smartphones. GPS world, 27(11), $43-48$.

Bertiger, W., Bar-Sever, Y., Dorsey, A., Haines, B., Harvey, N., Hemberger, D., Heflin, M., Lu, W., Moore, A.W., Murphy, D., Ries, P., Romans, L., Sibois, A., Sibthorpe, A., Szilagyi, B., Vallisneri, M., \& Pascal, W. (2020). GipsyX/RTGx, a new tool set for space geodetic operations and research. Advances in Space Research, 66(3), 469-489.

Chen, K., \& Gao, Y. (2005). Real-time precise point positioning using single frequency data. Proceedings of the 18th international technical meeting of the Satellite Division of The Institute of Navigation (ION GNSS 2005), 1514-1523.

Chen, B., Gao, C., Liu, Y., \& Sun, P. (2019). Real-time precise point positioning with a Xiaomi MI 8 android smartphone. Sensors, 19(12), 2835. 
Elmezayen, A., \& El-Rabbany, A. (2019). Precise point positioning using world's first dual-frequency GPS/GALILEO smartphone. Sensors, 19(11), 2593.

Erdoğan, B., Karlıtepe, F., Öcalan, T., \& Tunalığlu, N. (2018). Performance analysis of Real Time PPP for transit of Mercury. Measurement, 129, 358-367.

Estey, L. H., \& Meertens, C. M. (1999). TEQC: the multi-purpose toolkit for GPS/GLONASS data. GPS solutions, 3(1), 42-49.

Héroux, P., Gao, Y., Kouba, J., Lahaye, F., Mireault, Y., Collins, P., Macleod, K., Tétreault, P., \& Chen, K. (2004). Products and applications for Precise Point Positioning-Moving towards real-time. Proceedings of the 17th international technical meeting of the satellite division of The Institute of Navigation (ION GNSS 2004), 1832-1843.

Kulikov, R., Chugunov, A., \& Zamolodchikov, V. (2019). Investigation of collision warning possibilities by means of GNSS receivers of Android smartphones. IOP Conference Series: Materials Science and Engineering Vol. 695, No. 1, 12013.

Lipatnikov, L. A., \& Shevchuk, S. O. (2019). Cost Effective Precise Positioning with GNSS. The International Federation of Surveyors (FIG), No: 74.

Öcalan, T., Erdoğan, B., Tunalığlu, N., \& Durdağ, U. M. (2016). Accuracy investigation of PPP method versus relative positioning using different satellite ephemerides products near/under forest environment. Earth sciences research journal, 20(4), 1-9.

Pathak, V., Thornwall, S., Krier, M., Rowson, S., Poilasne, G., \& Desclos, L. (2003). Mobile handset system performance comparison of a linearly polarized GPS internal antenna with a circularly polarized antenna. IEEE antennas and propagation society international symposium. Digest. Held in conjunction with: USNC/CNC/URSI North American Radio Sci. Meeting Vol. 3, 666-669.

Robustelli, U., Baiocchi, V., \& Pugliano, G. (2019). Assessment of dual frequency GNSS observations from a Xiaomi Mi 8 Android smartphone and positioning performance analysis. Electronics, 8(1), 91.

Siddakatte, R., Broumandan, A., \& Lachapelle, G. (2017). Performance evaluation of smartphone GNSS measurements with different antenna configurations. Proceedings of the International Navigation Conference. 2007.

Takasu, T. (2013). RTKLIB ver. 2.4. 2 Manual. RTKLIB: An Open Source Program Package for GNSS Positioning, 29-49.

Wu, Q., Sun, M., Zhou, C., \& Zhang, P. (2019). Precise point positioning using dual-frequency GNSS observations on smartphone. Sensors, 19(9), 2189.

Zhang, X., Tao, X., Zhu, F., Shi, X., \& Wang, F. (2018). Quality assessment of GNSS observations from an Android N smartphone and positioning performance analysis using time-differenced filtering approach. GPS Solutions, 22(3), 1-11.

Zumberge, J. F., Heflin, M. B., Jefferson, D. C., Watkins, M. M., \& Webb, F. H. (1997). Precise point positioning for the efficient and robust analysis of GPS data from large networks. J. Geophys. Res., 102( B3), 5005- 5017

URL-1: GSA GNSS Market Report 2019, Issue6, https://www.gsa.europa.eu/newsroom/news/european-gnss-agency-gsa-releases-6thgnss-market-report, (Erişim Tarihi: 3 Şubat 2020)

URL-2: Geo++RINEX Logger, https://play.google.com/store/apps/details?id=de.geopp.rinexlogger\&hl=en, (Erişim Tarihi: 28 Ocak 2020). 